\title{
Experimental sensitivity analysis of a linearly stable thermoacoustic system via a pulsed forcing technique
}

\author{
Nicholas P Jamieson - Matthew P Juniper
}

Received: date / Accepted: date

\begin{abstract}
In this paper we present the results of an experimental sensitivity analysis on a vertical electricallyheated Rijke tube. We examine the shift in linear decay rates and frequencies of thermoacoustic oscillations, with and without control devices. To measure the decay rate we wait for the system to reach a steady state and then excite it with an acoustic pulse from a loudspeaker. We identify the range of amplitudes over which the amplitude decays exponentially with time. In this range, the rate of change of the amplitude is linearly proportional to the amplitude, and we calculate the constant of proportionality, the linear decay rate, which can be compared with model predictions. The aim of this work is (i) to improve the experimental techniques implemented by Rigas et al. (J. Fluid Mech., 2016, vol. 787, R1), Jamieson et al. (Int. J. Spray and Comb. Dyn., Accepted, 2016), using a technique inspired by Mejia et al. (Comb. and Flame, 169:287-296, 2016), and (ii) to provide experimental data for future comparison with adjoint-based sensitivity analysis. Our experimental set up is automated and we can obtain thousands of decay rates in 1/12 the time of our previous method.
\end{abstract}

Keywords Thermoacoustics · Acoustics · Flow Control · Dynamical Systems

\section{Introduction}

Thermoacoustic oscillations occur in many combustion chambers. They arise due to feedback between acoustic

\section{N. P. Jamieson}

Department of Engineering, University of Cambridge

E-mail: npj24@cam.ac.uk

M. P. Juniper

Department of Engineering, University of Cambridge waves and unsteady heat release rate, when the fluctuating heat release rate is sufficiently in phase with the unsteady pressure. The oscillations are characterised by large amplitude pressure fluctuations whose frequency is usually similar to the resonant acoustic modes of the combustion chamber. Thermoacoustic oscillations can compromise the safety, performance and reliability of combustion systems by increasing the mechanical vibration, thrust fluctuation, and thermal loading, which can even lead to complete system failure (Lieuwen and Yang, 2005). The suppression of thermoacoustic oscillations has also become important to the gas turbine industry because a shift to lean premixed engine operation conditions, to comply with legislation on reducing $N O_{x}$ emissions, has made these combustion systems more prone to thermoacoustic oscillations (Lieuwen and Zinn, 1998).

In this paper, we investigate the control of an electrically heated, vertical Rijke tube via three methods; (i) a passive drag device, (ii) a secondary heat source, and (iii) a variable area outlet. Using a technique inspired by Mejia et al. (2016), we repeat the measurements in Rigas et al. (2016) and Jamieson et al. (2016), which were for the passive drag device and secondary heat source, and perform new measurements for the variable area outlet. With this more efficient method, we compile large datasets of experimental results that are compared with theoretical predictions from adjointbased sensitivity analysis of models currently under development by the group.

The experiments are performed in the linearly stable regime, with control methods that alter the decay rate and frequency of oscillations. Our models and our previous experimental work (Rigas et al., 2016, Fig. $7 \mathrm{~b})$ show that, when passive control is implemented, the shift in growth rate when the system is unstable is 
equal and opposite to the shift in decay rate when the system is stable. In other words, passive control methods that make a stable system more stable also make an unstable system less unstable or stable. This means that these passive control methods will apply similarly to linearly unstable systems.

\subsection{Rijke tube and control}

The Rijke tube, first studied by Rijke (1859), is a simple configuration for the study of thermoacoustic instabilities (Raun et al., 1993). To explain the physical mechanism it is convenient to neglect the mean heat release rate from the heater and assume that the pressure fluctuation at the open ends of the tube is zero. Therefore, the fundamental acoustic mode has a pressure node at either end of the tube and an antinode at the midpoint. The acoustic velocity has a node at the centre and an antinode at either end. During the compression phase of the acoustic cycle, the acoustic velocity is directed towards the centre of the tube, causing the pressure in the tube to increase. When the heater is placed in the upstream half of the tube, the acoustic velocity and the mean velocity are in the same direction during the compression phase. This causes the air speed around the heater, and therefore the heat transfer, to be larger than average during the compression phase. Conversely, the heat transfer is lower than average during the expansion phase. Critically, there is also a time delay (Lighthill, 1954) between the velocity at the heater and the subsequent heat release. These two properties mean that, over an acoustic cycle, more heat release occurs during moments of higher pressure than occurs during moments of lower pressure, causing more mechanical work to be done by the gas expanding during the expansion phase than is needed to compress it during the compression phase (Rayleigh, 1878). This results in the amplitude of acoustic oscillations growing until the heat release rate perturbations saturate and the excess work is balanced through acoustic radiation, viscous losses and thermal losses.

There are two categories of control which can be used to suppress thermoacoustic oscillations: passive control and feedback control (McManus et al., 1993; Candel, 2002; Dowling and Morgans, 2005). Passive control can be implemented by changing the design of a system, or installing further devices, such as Helmholtz resonators and baffles, for example as in the design of the F1 engine of the Saturn V rocket: baffles were introduced to the injector plate during 2000 full-scale engine tests leading to the suppression of thermoacoustic oscillations (Culick, 1988). A sub-field of passive control is tuned passive control, in which the characteristics of the passive control methods are tuned in situ, for example by changing the power through a secondary heater $(\S 4)$ or modifying the throat area of the system outlet via a mechanical iris $(\S 5)$. Tuned passive control via a secondary heater has been described by Katto and Sajiki (1977) and Sreenivasan et al. (1985). In Katto and Sajiki (1977) it was shown that a secondary heater located at $x / L=0.75$ had a stabilising effect on the system when the primary heat source was located at $x / L=0.25$.

One of the earliest applications of feedback control in thermoacoustics was performed by Heckl (1988). In this study, a microphone was placed upstream of the heat source. The signal was phase-shifted, amplified and transmitted to a loudspeaker downstream. This simple feedback loop was able to suppress thermoacoustic oscillations in a Rijke tube. More recently, work on active control of thermoacoustic oscillations has been performed by Zhang et al. (2015) and Zhao et al. (2015).

\subsection{Adjoint-methods in thermoacoustics}

Adjoint-based sensitivity analysis was first introduced to thermoacoustic instability by Magri and Juniper (2013). In traditional sensitivity analysis, the eigenvalues are calculated at a single operating point, a parameter is then varied slightly, and the eigenvalues are re-calculated at the new operating point. This approach gives the sensitivity of all the eigenvalues to a change in a single parameter. In adjoint-based sensitivity analysis, the eigenvalues are calculated for one operating point. Then one eigenvalue is selected and the governing equations reformulated such that the second calculation gives the sensitivity of that eigenvalue to a change in all parameters. In thermoacoustics, usually only a few eigenvalues are of interest, but many parameters can be modified. Adjoint-based sensitivity analysis is therefore much more efficient than traditional sensitivity analysis. In addition, the sensitivities calculated with a single adjoint calculation can be combined such that the effect of any passive device can be determined with one further cheap calculation. Using this technique, Magri and Juniper (2013, 2014), were able to determine the effect a passive drag device and a secondary heater have on the shift in linear growth rate and shift in frequency of thermoacoustic oscillations. The sensitivity information obtained can be combined with optimization algorithms to develop optimal passive control strategies. It is important to note that this method is a linear technique, and therefore is only accurate for small changes to parameters. 


\subsection{Sensitivity analysis in thermoacoustics}

The first comparison of experimental sensitivity analysis with adjoint-based sensitivity analysis in thermoacoustics were by Rigas et al. (2016) and Jamieson et al. (2016). Rigas et al. (2016) developed an automated experiment to measure linear growth rates and decay rates for a range of primary heater powers, $P_{1}$, and axial locations of a passive drag device, $x_{c} / L$. They determined the shift in linear growth and decay rate due to the introduction of a passive drag device by comparing measurements obtained with and without the passive drag device installed in the system. The experimental results were compared with the theoretical predictions of adjoint-based sensitivity analysis finding good qualitative agreement for the shift in growth rate, but not the shift in frequency of oscillations observed during periods of growth (Magri and Juniper, 2013). The disagreement for the shift in frequency was due to the model of Magri and Juniper (2013) not accounting for a mean shift in flow temperature caused by the introduction of the passive drag device.

Jamieson et al. (2016) measured linear growth rates and decay rates for a range of secondary heater powers, $P_{2}$, and axial locations, $x_{c} / L$. The experimental process consisted of three steps: (i) the Rijke tube system was heated to a steady-state with $P_{1} \approx 138 \mathrm{~W}$, (ii) $P_{1}$ was abruptly increased to $\approx 393 \mathrm{~W}$ and the linear growth rate was measured as the system transitioned to a stable limit cycle, and (iii) $P_{1}$ was abruptly decreased to $\approx 138$ $\mathrm{W}$, and the linear decay rate was measured as the system transitioned to a stable fixed point. This procedure was repeated for a range of $P_{2}$ at $x_{c} / L=0.05$ to 0.20 and 0.30 to 0.95 . The experimental results were compared with the theoretical predictions of adjoint-based sensitivity analysis finding good qualitative agreement for the shift in growth rate (Magri and Juniper, 2013). A discrepancy in the frequency during periods of linear growth and decay exists for the same reasons as discussed above and highlights the limitations of the model used, reiterating the need for the experimental measurement of sensitivities to be used as a rigorous test of any thermoacoustic model.

Mejia et al. (2016) studied four experimental methods for extracting growth and decay rates on a methaneair laminar burner at a range of operating points. The methods were: (i) a harmonic response method in which a loudspeaker forced the flame in the linearly stable regime; (ii) a white noise method in which a stochastic signal was used to force the flame in either the linearly stable or unstable region; (iii) an impulse response method in which an impulse signal was used to momentarily excite the flame in the linearly stable regime, allowing for a decay rate to be measured as the system returns to a steady-state; and (iv) an active control method which involved switching on and off an active controller in the unstable regime and measuring the temporal evolution of the oscillations. Fittings were applied to power spectral density and time-series signals of acoustic pressure fluctuations, acoustic velocity fluctuations and heat release fluctuations, from which the growth rate and frequency were determined. It was found that all four methods were suitable for determining the growth rates, decay rates, and frequencies of thermoacoustic oscillations.

This paper builds on the work of Rigas et al. (2016), Jamieson et al. (2016) and Mejia et al. (2016) to develop a more efficient experimental technique for experimental sensitivity analysis in thermoacoustics. The pulsed forcing technique presented in this paper allows the same amount of data to be collected in approximately $1 / 12$ the time of previous work, thus allowing for significantly larger data sets to be obtained and used to refine thermoacoustic models.

\section{Experimental set up and data processing}

\subsection{Apparatus}

Experiments were conducted on a $1 \mathrm{~m}$ long stainless steel vertical Rijke tube with an internal diameter of $47.4 \mathrm{~mm}$ and a wall thickness of $1.7 \mathrm{~mm}$ (figure 2). For all experiments, the primary heat source was attached to two rods and held in place at $x_{p} / L=0.25$, the ideal location for the self-excitation of thermoacoustic oscillations (Saito, 1965). The primary heater (figure 1a) was powered by a 640 Watt EA Elektro-Automatik EA-PSI 5080-20 A DC programmable power supply. A G.R.A.S. 46 AG 1/2" LEMO microphone with a sensitivity of 12 $\mathrm{mV} / \mathrm{Pa}$ was used to measure the thermoacoustic oscillations. The microphone was angled at $45^{\circ}$ towards the inlet of the Rijke tube and placed approximately $55 \mathrm{~mm}$ from the bottom of the tube. The raw pressure signal was sampled at $10 \mathrm{kHz}$, much higher than the anticipated frequencies of the thermoacoustic oscillations, 180 - $190 \mathrm{~Hz}$. Type-K thermocouples were secured at the centreline of the inlet and outlet, and at $x / L$ $=0.05,0.25,0.50,0.75$, and 0.95. Temperature data were sampled continuously at $1 \mathrm{~Hz}$ and logged with an Omega TC-08 DAQ. A Pro Signal 55-1205 loudspeaker was fixed at the base of the tube. The loudspeaker was connected to an STA-500 $600 \mathrm{~W}$ Pro Power amplifier and provided an acoustic pulse controlled through National Instruments LabVIEW. All data was acquired through National Instruments BNC-2110 DAQ device using LabVIEW. 


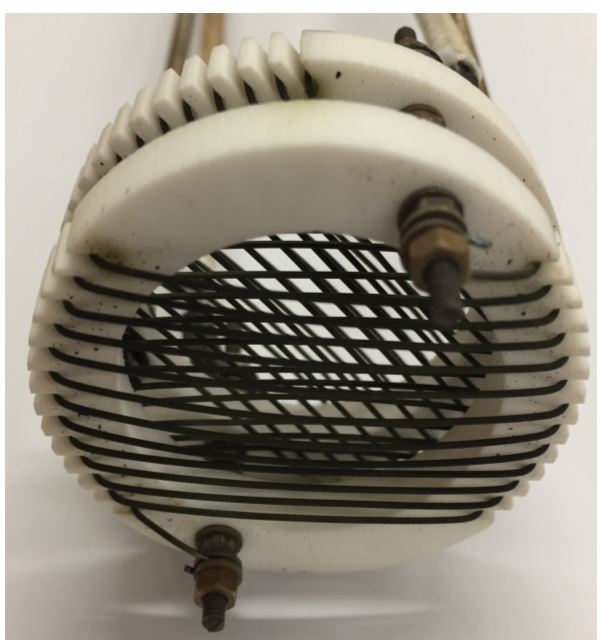

(a)

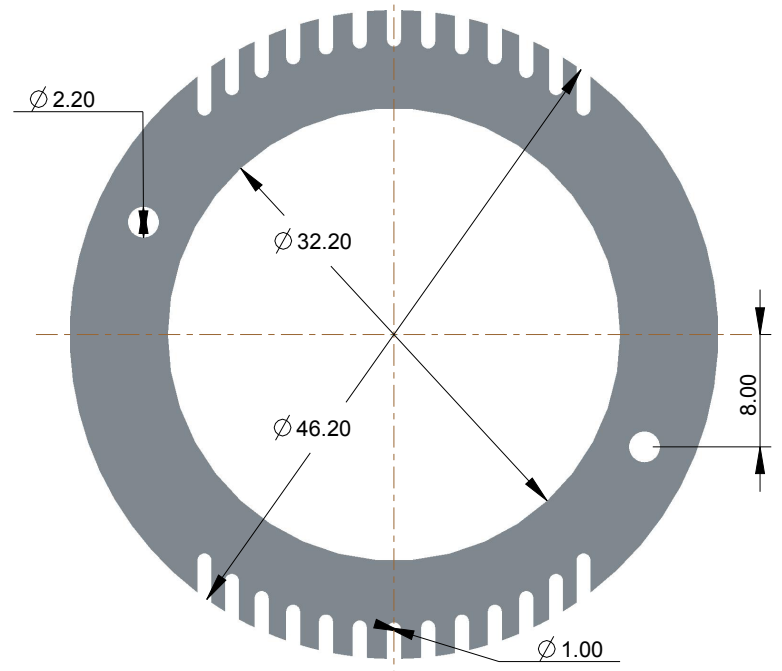

(c)

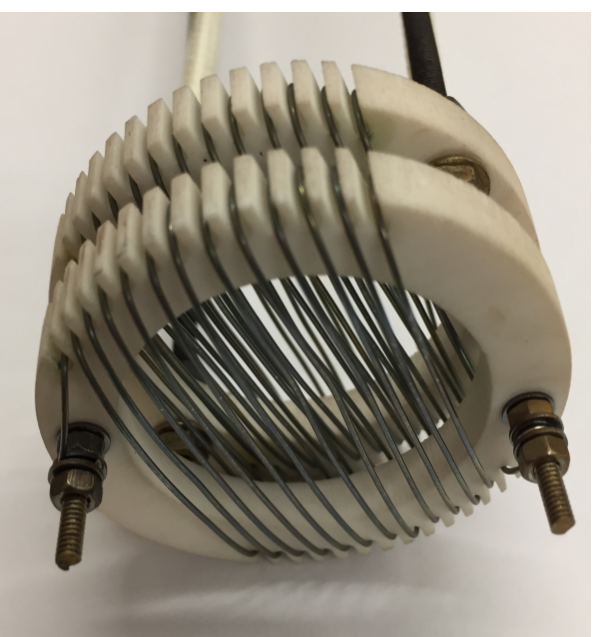

(b)

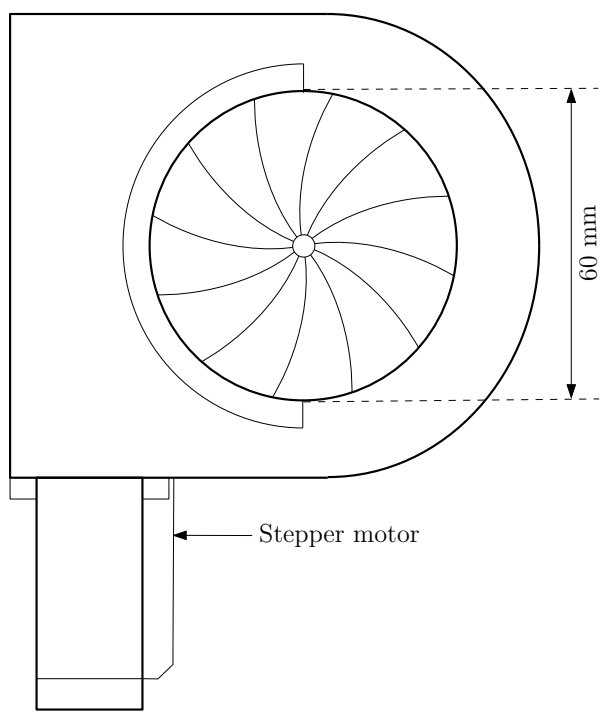

(d)

Fig. 1: (a) Primary heater. (b) Passive drag device and secondary heat source. (c) Top disk of (a) and (b). (d) Diagram of the variable cross-sectional area outlet (iris).

The set-up varied according to the control method being used: (i) for the passive drag device, a wire was wrapped around two ceramic discs (figure 1b) and mounted on two rods attached to an automated digital height gauge. This could be automatically traversed through the tube with an accuracy of $\pm 0.01 \mathrm{~mm}$; (ii) the secondary heat source used a ISO-TECH IPS $201020 \mathrm{~V}$ 10 A programmable power supply. The secondary heat source was also a wire wrapped around two ceramic discs (figure 1b). It was also mounted to two rods and attached to an automated digital height gauge, allowing it to be automatically traversed throughout the tube with an accuracy of $\pm 0.01 \mathrm{~mm}$; (iii) for the variable area outlet case, a Standa 8MID60-4-H motorised iris with a maximum aperture of $60 \mathrm{~mm}$ and a minimum aperture of $4 \mathrm{~mm}$ was mounted at the outlet of the Rijke tube (figure 1d). The iris was controlled via a Standa 8SMC4-USB-B8-1 1-axis stepper motor and 


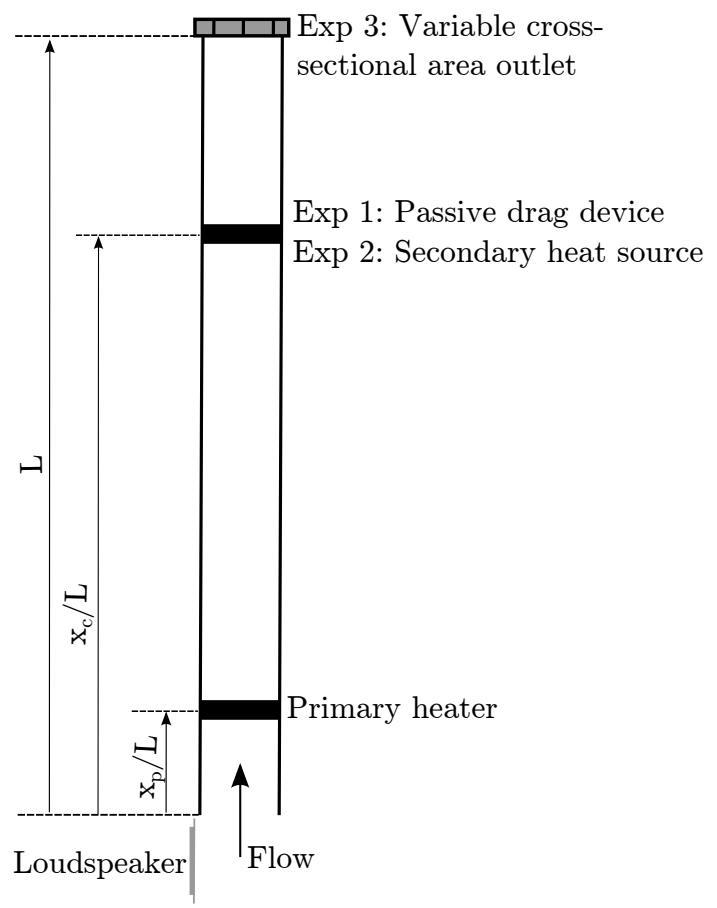

Fig. 2: Experimental set-up. Experiment 1: passive drag device and two open ends; Experiment 2: secondary heat source and two open ends; Experiment 3: variable area at the outlet of the tube.

could withstand temperatures of approximately $400^{\circ} \mathrm{C}$, much higher than those expected during the experimental campaign, approximately $250-300^{\circ} \mathrm{C}$.

Figure 1c shows a CAD drawing of the MACOR ceramic disks used in the heat source and passive drag device. The wire used to construct the primary heater, passive drag device and secondary heat source is $80-20$ Nickel Chrome Alloy. The wire had a thickness of 0.559 $\mathrm{mm}$, an estimated flow facing length of $670 \mathrm{~mm}$ per disk, and theoretical emissivity of 0.87-0.89 (OMEGA Engineering Inc, 2017). The mean flow velocity through the tube was not measured but is estimated to be between 0.1 and $1 \mathrm{~m} / \mathrm{s}$.

\subsection{Data acquisition and processing}

This Rijke tube exhibits the dynamical system behaviour of a sub-critical Hopf bifurcation. The acquisition of a single data point, containing both a linear decay rate and frequency of the oscillations during periods of linear decay, is shown in figure 3. Figure 3a shows the raw pressure signal of a single data point. The acquisition time was 10 seconds. During data acquisition the primary heat source had a constant power of 170 Watts. A simple proportional-integral controller was implemented to ensure that, if the resistance of the heater changed over the course of many hours, the power would remain constant, thus not introducing a systematic error into the baseline measurements.

In this study we set $P_{1}=170 \mathrm{~W}$, so that the system remained in the linearly stable region. An acoustic pulse was delivered by the loudspeaker after approximately 1.5 seconds. The acoustic pulse was a sinusoidal wave of $\approx 2.5 \mathrm{~Pa}$ amplitude, 0.05 seconds time duration, and $175 \mathrm{~Hz}$ frequency. This stimulated the first mode for around 9 cycles, which differs from (Mejia et al., 2016) who used a short Gaussian pulse. The type of forcing is unimportant, as long as there is sufficient amplitude to lead to a long period of linear decay in the mode one wishes to examine. As can be seen in figure [2], the amplitude caused by the initial pulse was larger than strictly required and there was a long period of linear decay shortly afterwards.

We applied a bandpass Butterworth filter to the raw pressure signal before using the Hilbert transform to determine the instantaneous amplitude, $A(t)$, and phase $\phi(t)$ of the signal in a similar framework to Provansal et al. (1987) and Schumm et al. (1994). In Matlab, the hilbert function determines an analytical signal of the form $X(t)=x(t)+i x_{H}(t)=A(t) e^{i \phi(t)}$, where $x(t)$ is the original signal, $A(t)$ and $\phi(t)$ have the same definition as above, and the Hilbert transform of $x(t)$ is $x_{H}(t)=\pi^{-1} P . V . \int_{-\infty}^{\infty} \frac{s(\tau)}{t-\tau} d \tau$, where P.V. indicates that the Cauchy principal value is taken. Whilst $A(t)$ and $\phi(t)$ can be computed via this method for any arbitrary $x(t)$, they only have a physical meaning if the signal is narrowband and has a dominant frequency peak (Pikovsky et al., 2003), as was the case for our signal. The filtered analytical signal had a noise-free region of linear decay between two thresholds (figure $3 \mathrm{~b}$ ). The upper and lower limits were set to ensure the linear fit was not influenced by the acoustic pulse signal or noise floor. The linear decay rates were given by $\sigma_{r}=$ $\mathrm{d}(\ln (A)) / \mathrm{d} t$ and the frequency during periods of linear decay were given as $\sigma_{i}=\mathrm{d}(\phi) / \mathrm{d} t$.

At each operating point in $\S 3, \S 4$ and $\S 5,150$ data points were obtained so that a detailed uncertainty quantification of the random error could be performed. The uncertainty analysis followed the process outlined in Jamieson et al. (2016) and all error bars are presented with a $95 \%$ confidence interval.

\section{Control via a passive drag device}

This section details an experimental sensitivity analysis that was performed to determine the effect of a passive drag device on the shift in linear decay rate and oscillation frequency during periods of linear decay. 


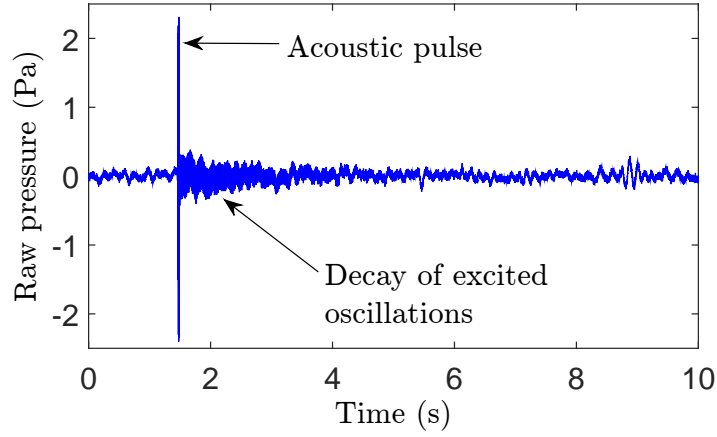

(a)

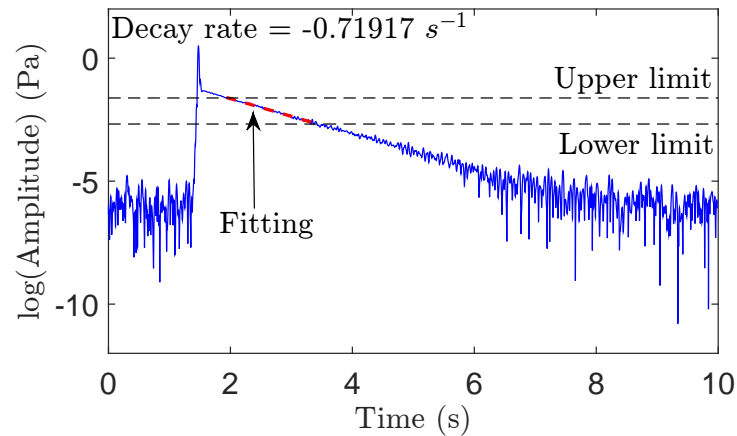

(b)

Fig. 3: (a) Raw pressure signal of single data point. (b) Filtered analytical signal obtained using the Hilbert transform. Red dashed line denotes the region of linear decay from which data was extracted.

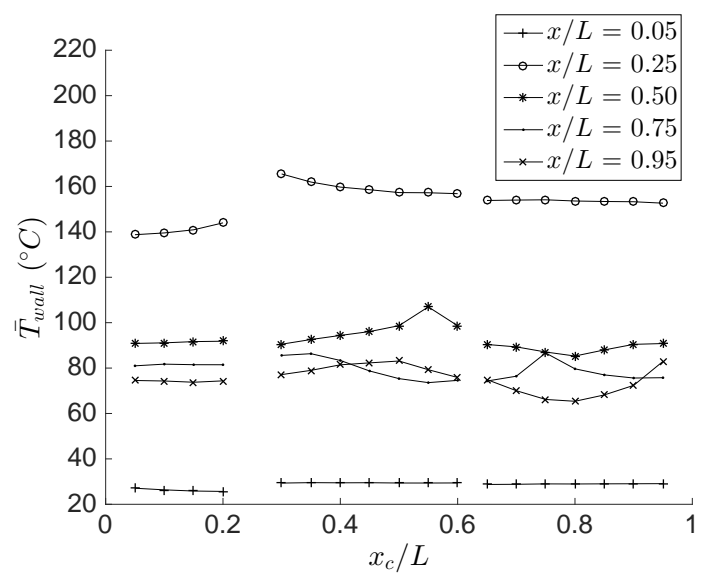

Fig. 4: Mean wall temperature, measured at position $x / L$, with the passive device placed at position $x_{c} / L$.

\subsection{Methodology}

The experimental method for this control case consisted of the following steps: (i) with no control device installed in the Rijke tube, the system was allowed to reach a steady-state with $P_{1}=170 \mathrm{~W}$; (ii) 150 acoustic pulses were given in 10 second intervals resulting in 150 baseline data points; (iii) the passive drag device was introduced at a specific axial location, $x_{c} / L$, and then 150 acoustic pulses were given in 10 second intervals resulting in 150 data points for a given $x_{c} / L$; (iv) step (iii) was repeated for each axial location, between $x_{c} / L=0.05$ to 0.20 and 0.30 to 0.95 ; (v) step (ii) was then repeated to determine whether the baseline measurements of the system had drifted over the course of the experiments. The final baseline was determined by averaging both the "before" baseline (step (ii)) and the "after" baseline (step (v)) and interpolating a 150 point linear spacing between the two, accounting for any drift in the baseline over the course of the experiment. Figure 4 shows the wall temperatures measured during these experiments.

\subsection{Shift in linear decay rate and oscillation frequency}

The linear decay rate, $\sigma_{r}$, and oscillation frequency during periods of linear decay, $\sigma_{i}$, were measured experimentally for a range of $x_{c} / L$. The shift in linear decay rate and oscillation frequency defined in the theoretical studies of Magri and Juniper (2013) were experimentally obtained as

$\delta \sigma_{r, i}\left(P_{1}, x_{c} / L\right)=\sigma_{r, i, c}\left(P_{1}, x_{c} / L\right)-\sigma_{r, i, 0}\left(P_{1}\right)$

where $\sigma_{r, c}$ is the linear decay rate measured with the passive drag device installed, $\sigma_{i, c}$ is the oscillation frequency with the passive drag device installed, $\sigma_{r, 0}$ is the baseline linear decay rate, and $\sigma_{i, 0}$ is the baseline oscillation frequency. The baseline linear decay rate and oscillation frequency were measured with only the primary heater installed. To determine the shift in linear decay rate, the data obtained for each $x_{c} / L$ was processed in an identical way. For brevity, only the data for $x_{c} / L=0.65,0.70,0.75,0.80,0.85,0.90$, and 0.95 is presented (figure 5 ).

Figure 5 a shows the baseline linear decay rates, $\sigma_{r, 0}$, and the linear decay rates when a passive drag device was installed, $\sigma_{r, c}$, for a range of $x_{c} / L$. As the passive drag device was traversed toward $x_{c} / L=0.95$, the magnitude of the measured linear decay rate increased, indicating that the system was becoming more stable. This is expected because, at this position, there is an antinode of both the velocity and the receptivity of the momentum equation (Magri and Juniper, 2013). It was also seen that as the passive drag device was traversed toward $x_{c} / L=0.95$ the variance in the data increased. 


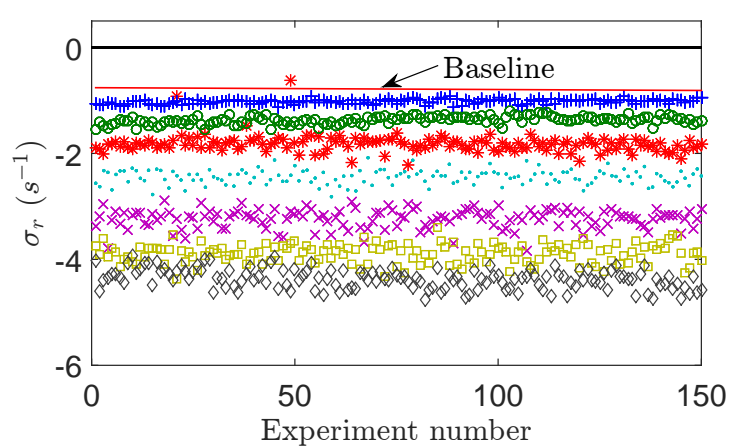

(a)

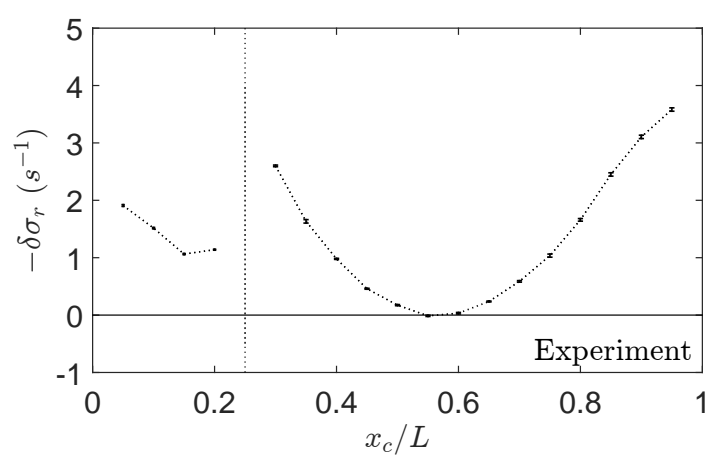

(c)

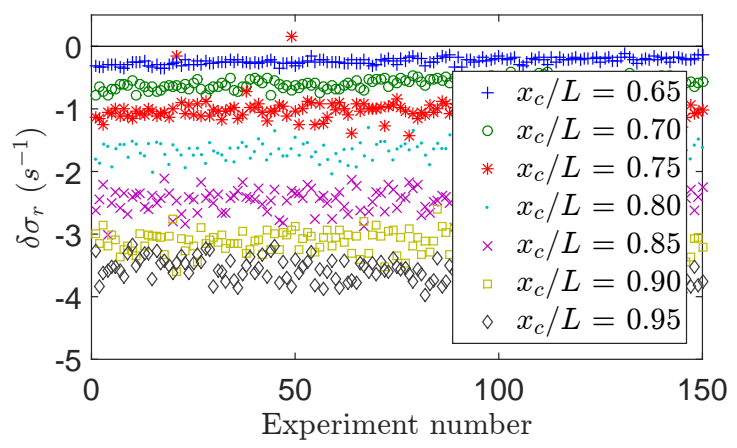

(b)

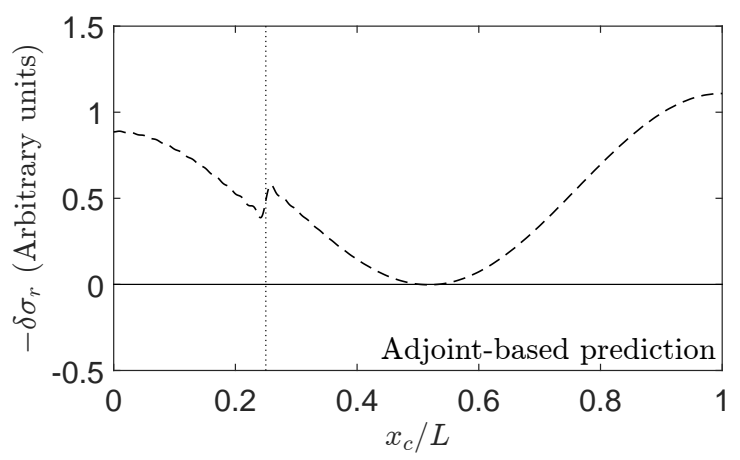

(d)

Fig. 5: (a) Baseline linear decay rate $\left(\sigma_{r, 0}\right)$, and controlled linear decay rate $\left(\sigma_{r, c}\right)$ for a range of passive drag device locations $\left(x_{c} / L\right)$. (b) The shift in linear decay rate $\left(\delta \sigma_{r}=\sigma_{r, c}-\sigma_{r, 0}\right)$ due to the passive drag device. (c) The shift in linear decay rate as a function of $x_{c} / L$. (d) Theoretical predictions of Magri and Juniper (2013). Note: the legend is identical for (a) and (b) and represents $x_{c} / L$ and all error bars are presented for a $95 \%$ confidence interval.

This was because at these more stable locations of the passive drag device, the linear decay rate was obtained from a fitting over fewer thermoacoustic oscillation cycles and therefore was more susceptible to random measurement error.

Figure $5 \mathrm{~b}$ shows the difference between $\sigma_{r, 0}$ and $\sigma_{r, c}$ for a range of $x_{c} / L$. The shift in linear decay rate due to the introduction of a passive drag device was then calculated by averaging the $\delta \sigma_{r}$ data obtained and plotting it as a function of $x_{c} / L$ (figure $5 \mathrm{c}$ ). It can be seen in figure $5 \mathrm{c}$ that the introduction of the passive drag device has a minimal effect when placed at $x_{c} / L=$ 0.60 and the largest stabilising effect when placed at $x_{c} / L=0.95$. In comparing figure $5 \mathrm{c}$ and $5 \mathrm{~d}$ it can be seen that the experimental results qualitatively agree well with the adjoint-based predictions of Magri and Juniper (2013) which did not attempt to give absolute values of the shifts.

Figure $6 \mathrm{a}$ shows the baseline oscillation frequency, $\sigma_{i, 0}$, and the oscillation frequency when a passive drag device was installed, $\sigma_{i, c}$, during periods of linear de- cay, for a range of $x_{c} / L$. It was observed that as the passive drag device was traversed from $x_{c} / L=0.65$ to 0.95 the measured oscillation frequency decreased and the variance in the data increased. Figure $6 \mathrm{~b}$ shows the difference between $\sigma_{i, 0}$ and $\sigma_{i, c}$ for a range of $x_{c} / L$. The shift in oscillation frequency was calculated in the same way as the shift in linear decay rate and is shown in (figure 6c). Comparing the figures $6 \mathrm{c}$ and $6 \mathrm{~d}$ it is clear that the shift in oscillation frequency predicted by Magri and Juniper (2013) does not agree with experimental results, the reasons for which are discussed in Rigas et al. (2016) and section 1.3 of this paper.

\section{Control via a secondary heat source}

This section details an experimental sensitivity analysis that measured the effect of a secondary heat source on the shift in linear decay rate and oscillation frequency during periods of linear decay. 


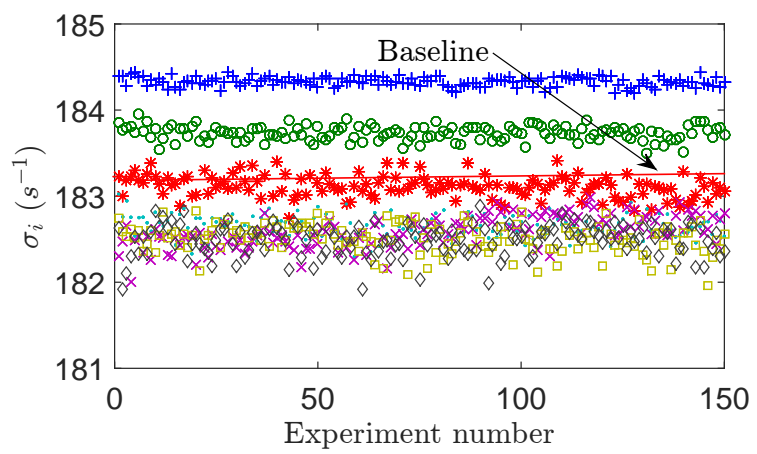

(a)

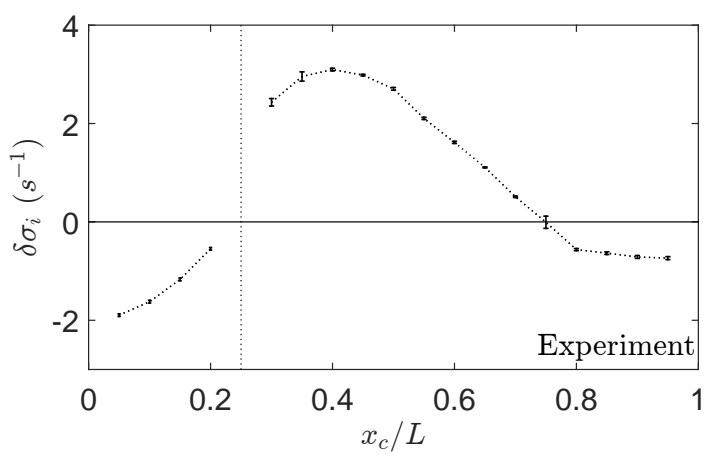

(c)

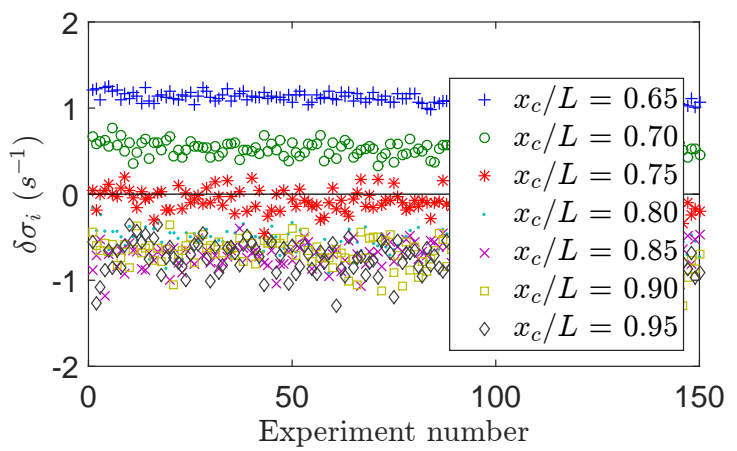

(b)

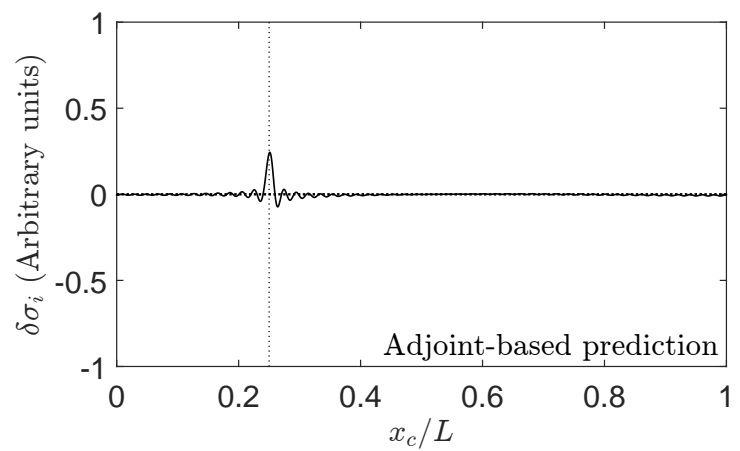

(d)

Fig. 6: (a) Baseline frequency during periods of linear decay $\left(\sigma_{i, 0}\right)$, and frequency during periods of linear decay with passive drag device installed $\left(\sigma_{i, c}\right)$ for a range passive drag device locations $\left(x_{c} / L\right)$. (b) The shift in frequency during periods of linear decay $\left(\delta \sigma_{i}=\sigma_{i, c}-\sigma_{i, 0}\right)$ due to the passive drag device. (c) The shift in frequency during periods of linear decay as a function of $x_{c} / L$. (d) Theoretical predictions of Magri and Juniper (2013). Note: the legend is identical for (a) and (b) and represents $x_{c} / L$ and all error bars are presented for a $95 \%$ confidence interval.

\subsection{Methodology}

The experimental method for this control case consisted of the following steps: (i) with the secondary heater installed at the first axial location, the system was allowed to reach a steady-state with $P_{1}=170 \mathrm{~W}$ and a secondary heat source power, $P_{2}=0 \mathrm{~W}$; (ii) at a given $x_{c} / L, 150$ acoustic pulses were given in 10 second intervals resulting in 150 baseline data points; (iii) at the same $x_{c} / L$ used in step (ii), $P_{2}$ was increased to $5 \mathrm{~W}$, at which point 150 acoustic pulses were given in $10 \mathrm{sec}-$ ond intervals resulting in 150 data points; (iv) step (iii) was repeated for $P_{2}=15,25,35$, and $45 \mathrm{~W}$, which resulted in four sets of 150 data points; (v) step (ii) was repeated twice, with $P_{2}=0 \mathrm{~W}$, allowing the system enough time to reach a steady-state. The second set of data was used as the second baseline measurement; (vi) steps (ii) - (v) were then repeated for $x_{c} / L=0.05$ to 0.20 and 0.30 to 0.95 .
4.2 Shift in linear decay rate and oscillation frequency

The linear decay rate, $\sigma_{r}$, and oscillation frequency during periods of linear decay, $\sigma_{i}$, were measured experimentally for a range of secondary heat source powers, $P_{2}$, and axial positions, $x_{c} / L$. The process used to measure the linear decay rates and oscillation frequency was discussed in $\S 4.1$ and was used for all data acquisition for this control case. The shift in linear decay rate and oscillation frequency, defined in the theoretical studies of Magri and Juniper (2013), were experimentally obtained as

$$
\begin{array}{r}
\delta \sigma_{r, i}\left(P_{1}, P_{2}, x_{c} / L\right)=\sigma_{r, i, c}\left(P_{1}, P_{2}, x_{c} / L\right)-\ldots \\
\sigma_{r, i, 0}\left(P_{1}, x_{c} / L\right)
\end{array}
$$

where $\sigma_{r, c}$ is the linear decay rate obtained with control, $\sigma_{i, c}$ is the oscillation frequency obtained with control, $\sigma_{r, 0}$ is the baseline linear decay rate obtained without control, $\sigma_{i, 0}$ is the baseline oscillation frequency obtained without control, $P_{1}$ and $P_{2}$ are the same as 


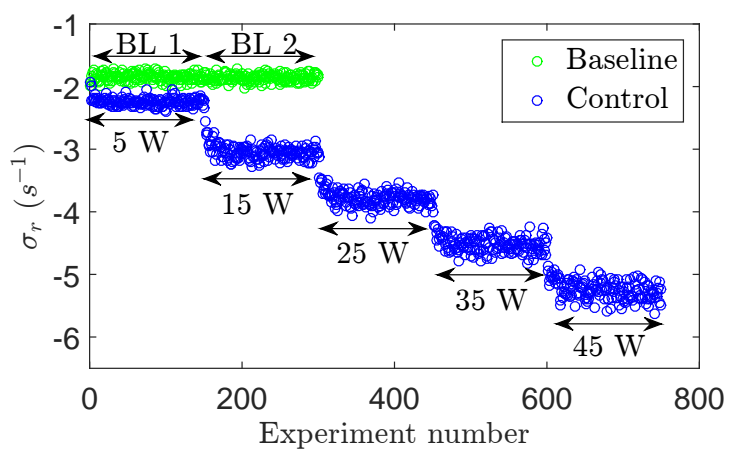

(a)

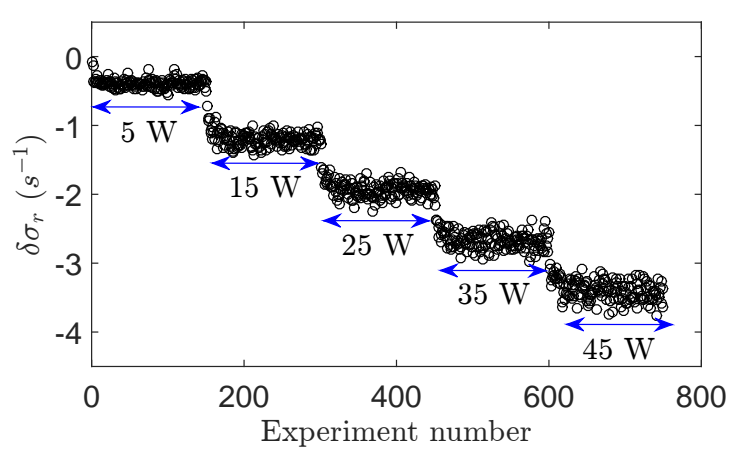

(c)

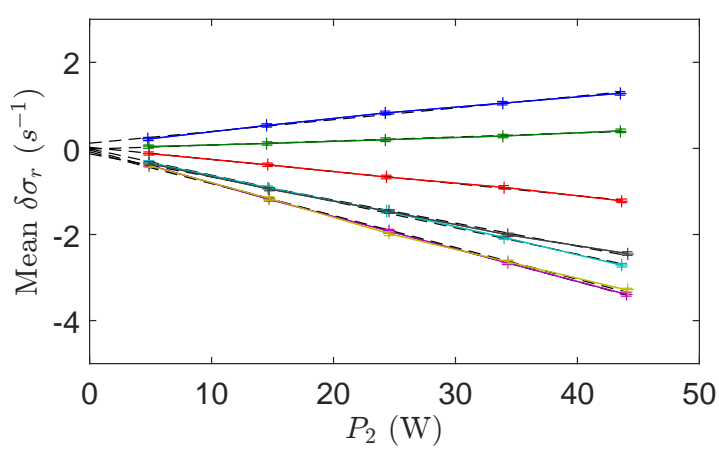

(e)

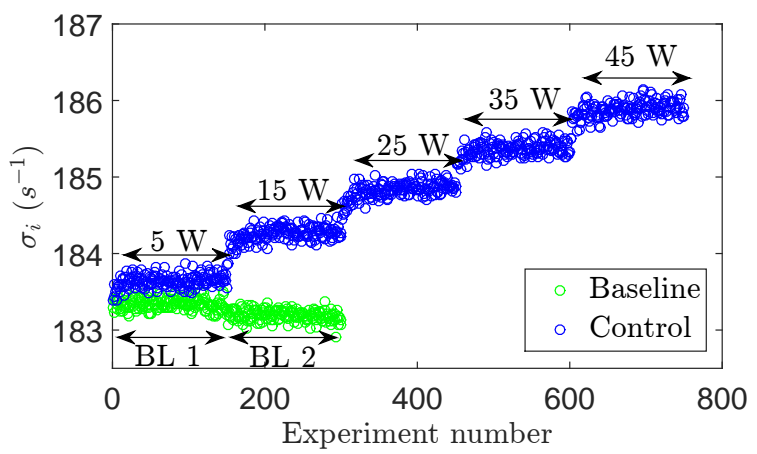

(b)

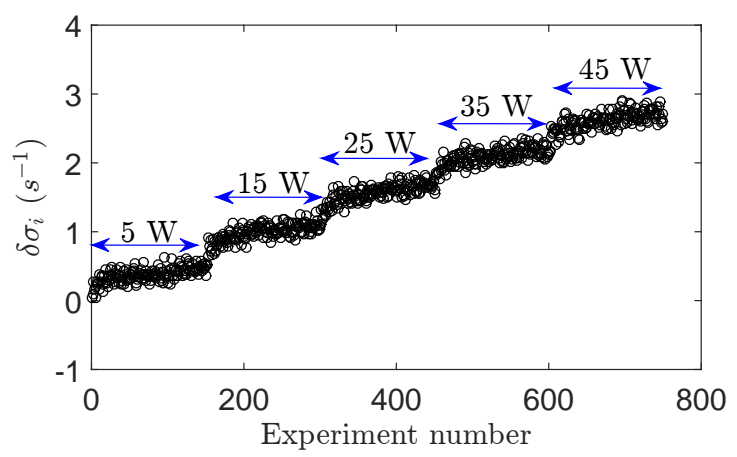

(d)

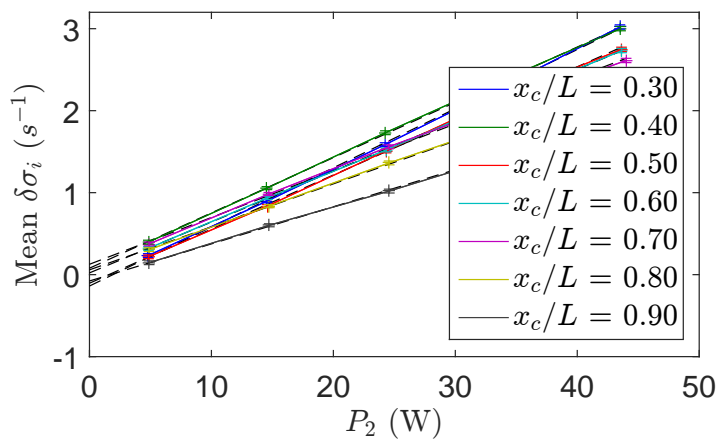

(f)

Fig. 7: (a) Linear decay rates measured with no control device $\left(\sigma_{r, 0}\right)$ and for a range of secondary heat source powers $\left(\sigma_{r, c}\right)$ at $x_{c} / L=0.70$. (b) Frequency during periods of linear decay measured with no control device $\left(\sigma_{i, 0}\right)$ and for a range of secondary heat source powers $\left(\sigma_{i, c}\right)$ at $x_{c} / L=0.70$. (c) Difference between the baseline linear decay rates and those measured for a range of secondary heater powers $\left(\delta \sigma_{r, c}\right)$ at $x_{c} / L=0.70$. (d) Difference between the baseline frequency during periods of linear decay and those measured for a range of secondary heater powers $\left(\delta \sigma_{i, c}\right)$ at $x_{c} / L=0.70$. (e) Averaged difference between the baseline linear decay rate $\left(\sigma_{r, 0}\right)$ and the linear decay rate obtained with a secondary heat source $\left(\sigma_{r, c}\right)$ for a range of $x_{c} / L$.(f) Averaged difference between the baseline frequency during periods of linear decay $\left(\sigma_{i, 0}\right)$ and the frequency during periods of linear decay obtained with a secondary heat source $\left(\sigma_{i r, c}\right)$ for a range of $x_{c} / L$. All error bars are presented with a $95 \%$ confidence interval. 


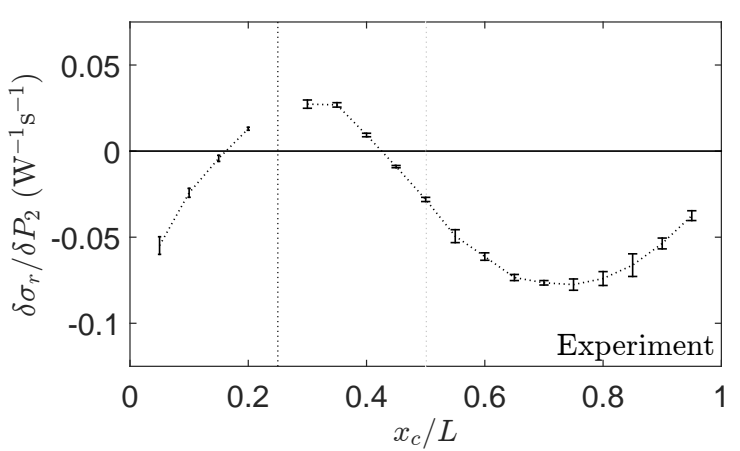

(a)

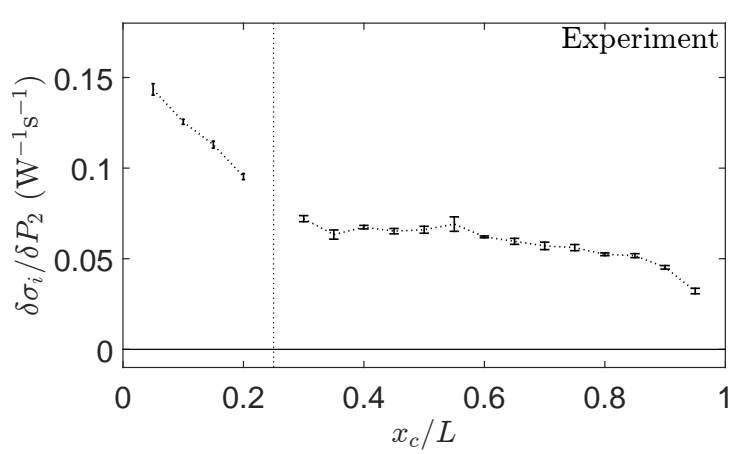

(c)

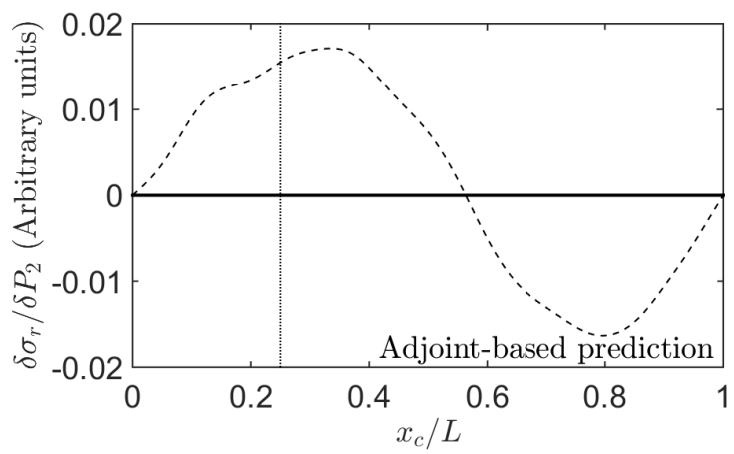

(b)

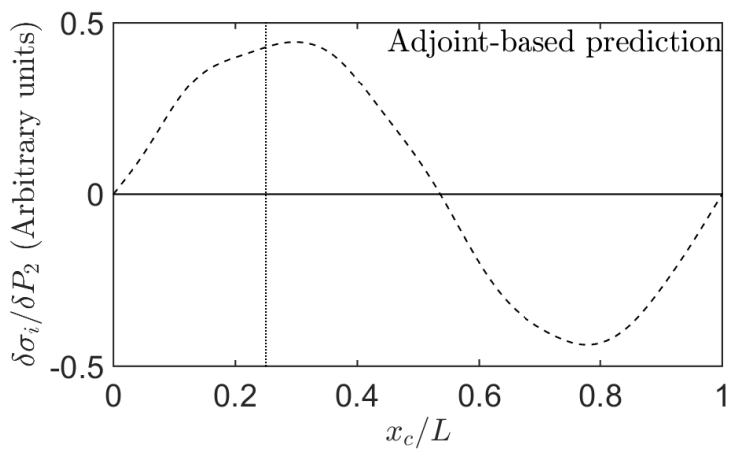

(d)

Fig. 8: (a) Shift in linear decay rate as a function of $x_{c} / L$. (b) Theoretical predictions of Magri and Juniper (2013). (c) Shift in frequency during periods of linear decay as a function of $x_{c} / L$. (d) Theoretical predictions of Magri and Juniper (2013). All error bars are presented for a $95 \%$ confidence interval.

previously defined. For brevity, only the data for $x_{c} / L$ $=0.70$ is presented here.

Figure 7a shows the baseline linear decay rates, $\sigma_{r, 0}$, and the linear decay rates measured in the presence of a secondary heat source, $\sigma_{r, c}$, for $x_{c} / L=0.70$. The final baseline was determined via the same process as discussed in $\S 3.1$. This 150 point dataset for the overall baseline was then subtracted from the corresponding dataset of each $P_{2}$ tested, resulting in the difference in linear decay rate with and without control, $\delta \sigma_{r}$ (figure $7 \mathrm{c})$.

It can be seen in figure 7a that, as the power of the secondary heat source was increased from $P_{2}=5$ $\mathrm{W}$ to $45 \mathrm{~W}$, the measured linear decay rate increased in magnitude. This increase in magnitude shows that, as expected, the secondary heat source at this location stabilizes the system.

Similar datasets to figure $7 \mathrm{a}$ and $7 \mathrm{c}$ were acquired at $x_{c} / L=0.05$ to 0.20 and 0.30 to 0.95 . Following this, each set of $\delta \sigma_{r}$ data was averaged for each individual $P_{2}$ tested. Figure 7e shows $\delta \sigma_{r}$ for $x_{c} / L=0.30,0.40,0.50$, $0.60,0.70,0.80$, and 0.90 , illustrating how the gradient of $\delta \sigma_{r}$ varies with $P_{2}$ and $x_{c} / L$. A linear fit was used to extract the gradient of the averaged $\delta \sigma_{r}$ data. It can be seen in figure $7 \mathrm{e}$ that, as the secondary heat source was traversed through the tube, the gradient decreased from $x_{c} / L=0.30$ to 0.70 and then increased from $x_{c} / L$ $=0.80$ to 0.90 .

The gradients obtained in figure 7e were then plotted as a function of $x_{c} / L$ to show the shift in linear decay rate due to the introduction of a secondary heat source (figure 8a). Figure $8 \mathrm{a}$ shows the results of the experimental sensitivity analysis which determined the shift in the linear decay rate due to the introduction of a secondary heat source. The experimental results are compared with the theoretical predictions by Magri and Juniper (2013) (figure 8b). It can be seen that the experimental results are shifted down on the vertical axis when compared with the theoretical predictions. The reason for this is not yet known.

Figure 7b shows the baseline oscillation frequency, $\sigma_{i, 0}$, and the oscillation frequency measured during periods of linear decay in the presence of a secondary heat source, $\sigma_{i, c}$, for $x_{c} / L=0.70$. The data was processed in the same way as discussed above and resulted in 
the difference in oscillation frequency with and without control, $\delta \sigma_{i}$ (figure $7 \mathrm{~d}$ ).

It can be seen in figures $7 \mathrm{~b}$ and $7 \mathrm{~d}$ that as $P_{2}$ was increased from $P_{2}=5 \mathrm{~W}$ to $45 \mathrm{~W}$, the measured oscillation frequency during periods of linear decay increases in magnitude and is always positive. This was expected because, by increasing $P_{2}$, the heat transfer from the heater to the mean flow increases. As the mean flow temperature increases, the local speed of sound increases, resulting in an increase in oscillation frequency that will always be positive.

Similar figures to figure $7 \mathrm{~b}$ and $7 \mathrm{~d}$ were acquired at $x_{c} / L=0.05$ to 0.20 and 0.30 to 0.95 and the same process as discussed for the linear decay rates was applied. Figure $7 \mathrm{f}$ shows $\delta \sigma_{i}$ for $x_{c} / L=0.30,0.40,0.50,0.60$, $0.70,0.80$, and 0.90 . Figure $7 \mathrm{f}$ demonstrates that as the secondary heat source was traversed through $x_{c} / L=$ 0.3 to 0.90 , the gradient decreased, but always remained positive.

The gradients obtained in figure $7 \mathrm{f}$ were plotted as a function of $x_{c} / L$ to show the shift in oscillation frequency observed during periods of linear decay due to the introduction of a secondary heat source (figure 8c). Comparing the figures $8 \mathrm{c}$ and $8 \mathrm{~d}$ it is clear that the shift in oscillation frequency predicted by Magri and Juniper (2013) again does not agree with experimental results. This physical behaviour was not seen in Magri and Juniper (2013) because their model does not take into account base flow changes caused by an increase in mean flow temperature, as mentioned in section 1.3.

\section{Control via a variable area outlet}

This section details an experimental sensitivity analysis that was performed to ascertain the effect of a variable area outlet (VAO), physically implemented with a motorised iris, on the shift in linear decay rate and oscillation frequency during periods of linear decay. To complement this data, a discussion on the effect of the $\mathrm{VAO}$ on the flow and wall temperature is also presented in $\S 5.3$.

\subsection{Methodology}

The experimental method for this control case consisted of the following steps: (i) with the VAO completely open, the system was allowed to reach a steady-state with a $P_{1}$ of $170 \mathrm{~W}$; (ii) with the VAO completely open, 150 acoustic pulses were given in 10 second intervals resulting in 150 baseline data points; (iii) the diameter, $D_{\text {iris }}$, of the VAO was set to $45 \mathrm{~mm}$ and 150 acoustic pulses were given in 10 second intervals resulting in 150 data points; (iv) step (iii) was repeated for $D_{\text {iris }}=40$ $\mathrm{mm}, 35 \mathrm{~mm}, 30 \mathrm{~mm}, 25 \mathrm{~mm}, 20 \mathrm{~mm}, 15 \mathrm{~mm}$, and 10 $\mathrm{mm}$; (v) step (ii) was repeated resulting in a second set of 150 baseline data points. The final baseline was determined via the same process as discussed in $\S 3.1$.

\subsection{Shift in linear decay rate and oscillation frequency}

The linear decay rates were measured experimentally for a range of VAO diameters, $D_{\text {iris }}$. The experimental process used to measure the linear decay rate was given in $\$ 5.1$, and was used for all data acquisition for this control case. The shift in linear decay rate and oscillation frequency was obtained experimentally as

$\delta \sigma_{r, i}\left(P_{1}, D_{\text {iris }}\right)=\sigma_{r, i, c}\left(P_{1}, D_{\text {iris }}\right)-\sigma_{r, i, 0}\left(P_{1}\right)$

where $P_{1}$ and $D_{\text {iris }}$ have the same definition as in $\S 5.1$. Data was acquired for $D_{\text {iris }}=45 \mathrm{~mm}, 40 \mathrm{~mm}, 35 \mathrm{~mm}$, $30 \mathrm{~mm}, 25 \mathrm{~mm}, 20 \mathrm{~mm}, 15 \mathrm{~mm}$ and $10 \mathrm{~mm}$. Figure 9a and $9 \mathrm{~b}$ show the shift in linear decay rate and shift in oscillation frequency observed during periods of linear decay, respectively, due to the VAO.

In figure $9 \mathrm{a}$ it can be seen that as the VAO was closed towards $D_{\text {iris }} / D_{\text {tube }}=0.2$ the linear decay rate increased. A second point of interest was that as the VAO was closed towards $D_{\text {iris }} / D_{\text {tube }}=0.2$, the variance in the obtained data increased. Because the closing of the VAO had such a stabilising effect on the system, the decay of oscillations was much faster when the aperture of the VAO was small. This fast decay meant that the linear decay rate was being calculated from a fitting over fewer thermoacoustic oscillation cycles resulting in greater variance in the observed data. In figure $9 \mathrm{~b}$ it can be seen that as the VAO diameter was reduced, the oscillation frequency continued to decrease until $D_{\text {iris }}=$ $10 \mathrm{~mm}$. This is probably because (figure 10a) the temperature of the gas in the tube drops as the iris is closed, for the reason described in the next section.

\subsection{Effect of outlet diameter on flow and wall temperature}

An array of type-K thermocouples were used throughout the experiment to record the effect that the VAO diameter had on the mean flow and wall temperature. This section examines: (i) the effect of the VAO on the inlet and outlet flow temperature for a range of $D_{\text {iris }}$; (ii) the effect of the VAO on the wall temperature at $x / L=0.05,0.25,0.50,0.75$, and 0.95 for a range of $D_{\text {iris. }}$.

Reducing $D_{\text {iris }} / D_{\text {tube }}$ restricts the flow and reduces the volumetric flow rate. Figure 10 shows (a) the mean 


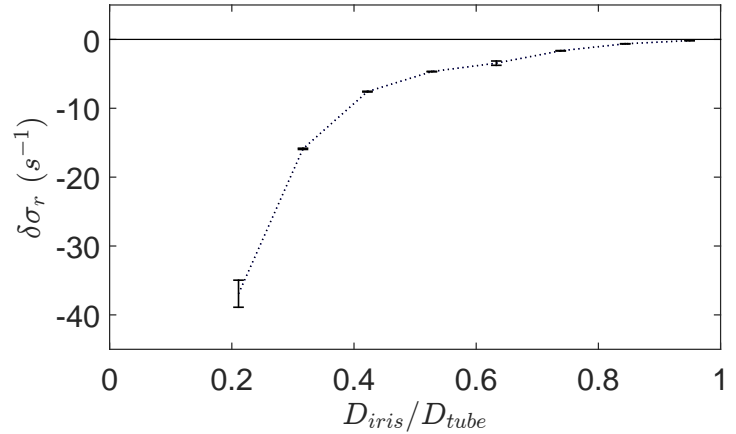

(a)

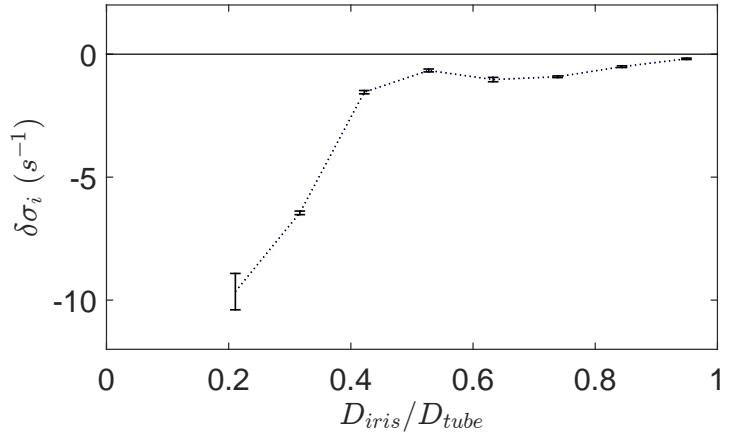

(b)

Fig. 9: (a) The shift in linear decay rate as a function of $D_{\text {iris }} / D_{\text {tube }}$. (b) The shift in frequency during periods of linear decay as a function of $D_{\text {iris }} / D_{\text {tube }}$.

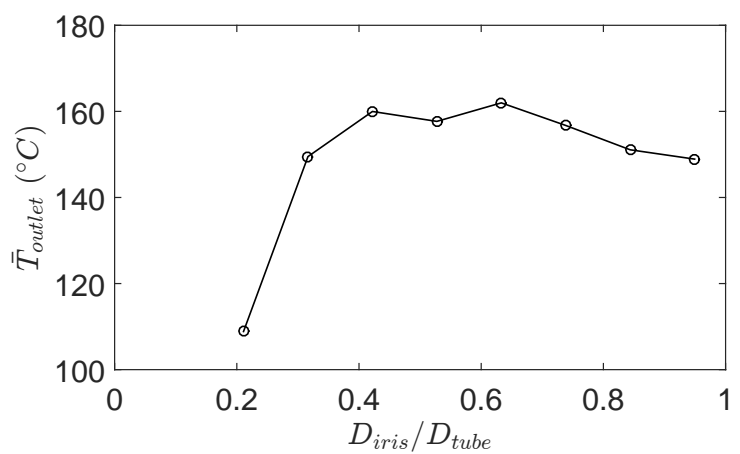

(a)

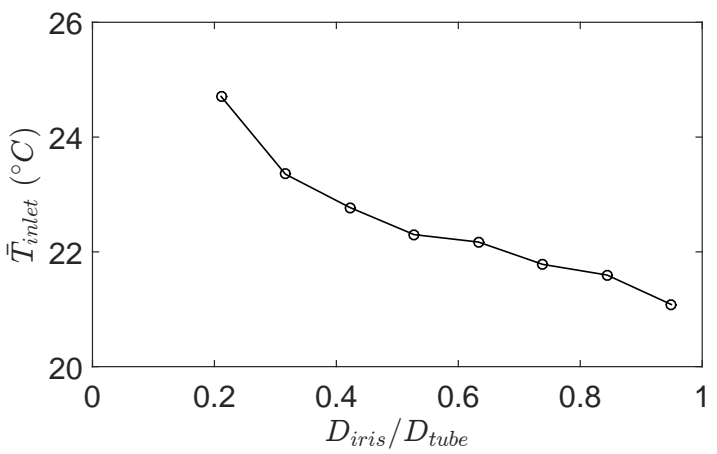

(b)

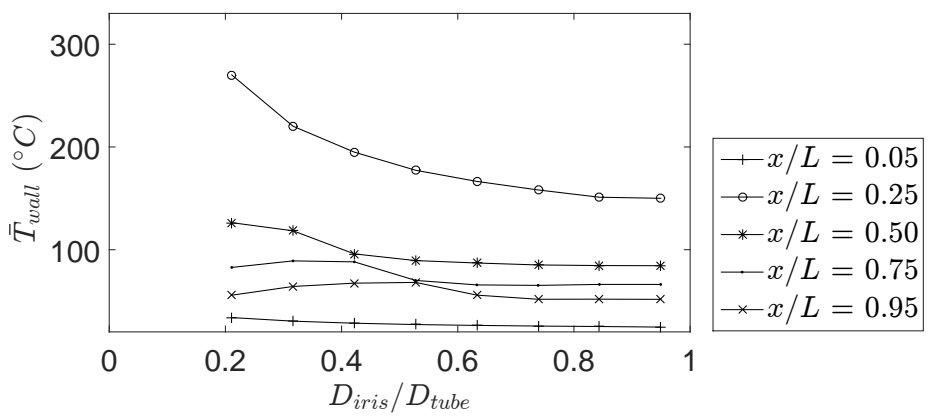

(c)

Fig. 10: (a) Mean outlet temperature measured at the centreline as a function of $D_{\text {iris }} / D_{\text {tube }}$. (b) Mean inlet temperature measured at the centreline as a function of $D_{\text {iris }} / D_{\text {tube }}$. (c) Mean wall temperature measured on outside of tube as a function of $D_{\text {iris }} / D_{\text {tube }}$. Note that all data points in (a), (b) and (c) are averaged over 150 experimental tests.

outlet temperature measured at the centre of the iris aperture, (b) the mean inlet temperature, and (c) the mean wall temperatures, $T_{w}$, as a function of $D_{\text {iris }} / D_{\text {tube }}$ at fixed heater power. These figures reveal two competing effects. On the one hand, reducing the volumetric flow rate at a fixed heater power increases the temperature jump across the heater. This effect is re- vealed in the monotonic increase of $T_{w}(x / L=0.25)$ as $D_{\text {iris }} / D_{\text {tube }}$ decreases in figure $10 \mathrm{c}$. On the other hand, reducing the volumetric flow rate increases the residence time of the gas in the Rijke tube and thereby increases the temperature drop due to heat loss through the walls. This effect is revealed through the fact that $T_{w}$ towards the top of the tube $(x / L=0.75$ and 0.95$)$ 
decreases rapidly when $D_{\text {iris }} / D_{\text {tube }}$ is reduced below around 0.5 , despite the fact that $T_{w}$ towards the middle of the tube $(x / L=0.25$ and 0.50$)$ increases due to the first effect. This shows that heat loss through the walls of the tube must be modelled if an iris is attached to the end of the Rijke tube.

\section{Conclusions}

The control of thermoacoustic oscillations is a perpetual problem for rocket and gas-turbine engine manufacturers. Previous work by Magri and Juniper (2013) has shown that adjoint-based sensitivity analysis could be a computationally efficient method by which optimal passive control strategies for the suppression of thermoacoustic oscillations can be developed. They rely, however, on accurate models. The aim of this study is to provide the data that can lead to the generation of accurate models.

In this paper, inspired by Mejia et al. (2016), we implement experimental sensitivity analysis significantly more efficiently that Rigas et al. (2016) and Jamieson et al. (2016). This technique, based on pulsed forcing, can collect thousands of data points in approximately $1 / 12$ the time of previous work. In parallel with the extension of previous work, we also provide a large amount of experimental data for future comparison with adjoint-based sensitivity analysis.

\section{References}

Candel, S. M. (2002). Combustion dynamics and control: Progress and challenges. Proc. Combust. Inst., $29: 1-28$.

Culick, F. E. C. (1988). Combustion Instabilities in Liquid-Fuelled Propulsion Systems. Technical Report 430.

Dowling, A. P. and Morgans, A. S. (2005). Feedback Control of Combustion Oscillations. Annu. Rev. Fluid Mech., 37(1):151-182.

Heckl, M. A. (1988). Active control of the noise from a rijke tube. J. Sound Vib., 124(1):117-133.

Jamieson, N. P., Rigas, G., and Juniper, M. P. (2016). Experimental sensitivity analysis via a secondary heat source in an oscillating thermoacoustic system. Int. J. Spray and Comb. Dyn., Accepted:1-10.

Katto, Y. and Sajiki, A. (1977). Onset of oscillation of a gas-column in a tube due to the existence of heatconduction field: a problem of generating mechanical energy from heat. Bulletin of JSME, 20(147):11611168 .
Lieuwen, T. and Zinn, B. T. (1998). The role of equivalence ratio oscillations in driving combustion instabilities in low NOx gas turbines. Symp. (Intl.) on Comb., 27(2):1809-1816.

Lieuwen, T. C. and Yang, V. (2005). Combustion instabilities in gas turbine engines: operational experience, fundamental mechanisms and modeling. Prog. in Astro. and Aero.

Lighthill, M. J. (1954). The Response of Laminar Skin Friction and Heat Transfer to Fluctuations in the Stream Velocity. Proc. of the Royal Society of London. Series A, Mathematical and Physical Sciences, 224(1156):1-23.

Magri, L. and Juniper, M. P. (2013). Sensitivity analysis of a time-delayed thermo-acoustic system via an adjoint-based approach. J. Fluid Mech., 719:183-202.

Magri, L. and Juniper, M. P. (2014). Adjoint-based linear analysis in reduced-order thermo-acoustic models. Int. J. Spray Combust. Dyn., 6(3):225-246.

McManus, K. R., Poinsot, T., and Candel, S. M. (1993). A review of active control of combustion instabilities. Prog. Energy Combust. Sci., 19:1-29.

Mejia, D., Miguel-Brebion, M., and Selle, L. (2016). On the experimental determination of growth and damping rates for combustion instabilities. Comb. and Flame, 169:287-296.

OMEGA Engineering Inc (Accessed May 2017). Transactions in Measurement and Control: Volume 1, Second Edition, Non-Contact Temperature Measurement. https://www.omega.com/literature/ transactions/transactions_Vol_I.pdf.

Pikovsky, A., Rosenblum, M., and Kurths, J. (2003). Synchronization: A Universal Concept in Nonlinear Sciences. Cambridge Nonlinear Science Series. Cambridge University Press.

Provansal, M., Mathis, C., and Boyer, L. (1987). Bénard-von Kármán instability: transient and forced regimes. J. Fluid Mech., 182:1-22.

Raun, R. L., Beckstead, M. W., Finlinson, J. C., and Brooks, K. P. (1993). A Review of Rijke Tubes, Rijke Burners and Related Devices. Prog. Energ. Combust., 19:313-364.

Rayleigh, J. W. S. (1878). The Explanation of Certain Acoustical Phenomena. Nature (London), 18:319321.

Rigas, G., Jamieson, N. P., Li, L. K. B., and Juniper, M. P. (2016). Experimental sensitivity analysis and control of thermoacoustic systems. J. Fluid Mech., 787(R1):1-11.

Rijke, P. L. (1859). On the vibration of the air in a tube open at both ends. Phil. Mag., 17:419-422.

Saito, T. (1965). Vibrations of Air-Columns Excited by Heat Supply. The Japan Society of Mechanical 
Engineers, 8(32).

Schumm, M., Berger, E., and Monkewitz, P. A. (1994). Self-excited oscillations in the wake of twodimensional bluff bodies and their control. J Fluid Mech., 271:17-53.

Sreenivasan, K., Raghu, S., and Chu, B. (1985). The control of pressure oscillations in combustion and fluid dynamical systems. In 1985 AIAA Shear Flow Control Conf.

Zhang, Z., Zhao, D., Han, N., Wang, S., and Li, J. (2015). Control of combustion instability with a tunable Helmholtz resonator. Aerosp. Sci. Technol., 41:55-62.

Zhao, D., Ji, C., Li, X., and Li, S. (2015). Mitigation of premixed flame-sustained thermoacoustic oscillations using an electrical heater. Int. J. Heat Mass Tran., 86:309-318. 